\section{Troubleshooting Forum}

\section{Molecular Biology Techniques Q\&A}

\section{Western blot: protein migration}

This month's question from the Molecular Biology Forums (online at molecularbiology.forums. biotechniques.com) comes from the "Protein Methods" section. Entries have been edited for concision and clarity. Mentions of specific products and manufacturers have been retained from the original posts, but do not represent endorsements by, or the opinions of, BioTechniques.

\section{Why doesn't my protein band appear at the correct apparent molecular weight? (Thread 19829)}

Q I am using Western blotting to look at a membrane channel of approximately $150 \mathrm{kDa}$ in size. I would like to confirm cross-reactivity of the antibody between human, mouse, and ferret. Each time I do the Western blot, I see a clear, reproducible, concentration-dependent band from each species, which confirms cross-reactivity. My problem is that the band is about $40 \mathrm{kDa}$ smaller than expected; no band appears at all in the expected position. If I pre-incubate the antibody for a negative control, the band vanishes. So I'm sure the labeling is coming from the primary antibody rather than from non-specific binding of the secondary antibody.

The samples were denatured for $5 \mathrm{~min}$ at $95^{\circ} \mathrm{C}$ with beta-mercaptoethanol and then stored for six months at $-80^{\circ} \mathrm{C}$ or 4 weeks at $-20^{\circ} \mathrm{C}$. Could either of those treatments result in protein cleavage? Or is it possible that the Kaleidoscope pre-stained marker shows a misleading pattern? I am using 12\% SDS, wet transferring overnight at 30V, and performing overnight blots with the primary antibody and non-specific controls while I am troubleshooting the procedure. The prestained marker seems to transfer fine, and Ponceau staining of the membrane shows a reasonable amount of protein throughout the lane. I have ordered another ladder to test, but could use any additional suggestions for solving this problem.

A Are there any confirmed or predicted glycosylation sites in the human primary amino acid sequence? If so, are those sites conserved in the ferret sequence? It is possible that the protein is processed differently in ferret cells. Instead of buying a new ladder, you can test the current one by blotting for another protein that runs around $150 \mathrm{kDa}$. Then compare the migration of the ladder to see if it looks correct. Have you cloned the ferret cDNA? If so, you may try epitope tagging it and expressing it in a human cell line to see where it runs. You should try the antibody against the protein along with an antibody against the tag. It would be beneficial to do the same experiment in a ferret cell line if you have one.

A You might be seeing a regulatory splice variant. Try RT-PCR to look for mRNA of different lengths.

A Is your antibody monoclonal?

A A Qiagen support document I have says: "SDS-PAGE is not a reliable method for accurately determining the molecular weight of proteins. This is why the term 'apparent molecular weight' is often used. The molecular weight estimated by SDS-PAGE can be very different from the actual molecular weight. Separation of proteins by SDS-PAGE depends on the uniform binding of negatively charged SDS to the protein to give a constant charge-to-mass ratio but, in reality, the amount of SDS bound can vary for different proteins."

You may be seeing the correct protein, but with the apparent molecular weight affected by hydrophobicity, charge, glycosylation, phosphorylation, or simply the amino acid sequence.

A Proteins often migrate differently from their predicted molecular mass, but $40 \mathrm{kDa}$ is a big shift. To begin, you should consult the data sheet that came with the antibody. How big is the antibody? Did the company provide an image of a sample Western blot?

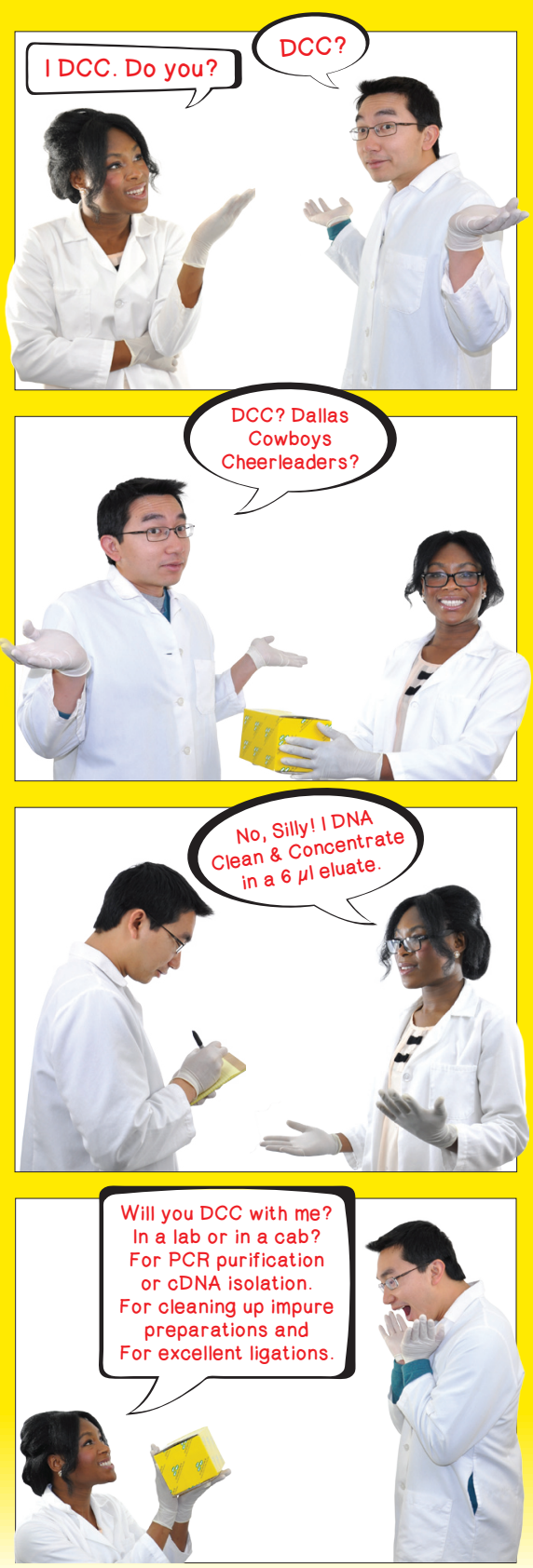

DNA Clean \& Concentrator" (DCC ${ }^{\mathrm{TH}}$ )

\section{DNA Clean-up from Diverse Sources}

$\checkmark$ PCR, restriction digests, RT reactions, enzyme removal, desalting, etc.

$\checkmark$ Genomic DNA clean-up

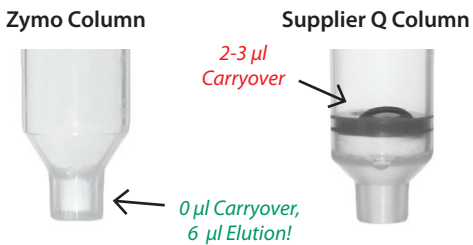

Get your DCC'" sample at www.zymoresearch.com/DCC

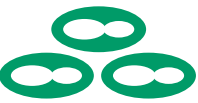

ZYMO RESEARCH

The Beauty of Science is to Make Things Simple 


\section{Effortless Imaging}

GelMax ${ }^{\mathrm{TM}}$ Imager

Benchtop solution

simplifies

precast and

mini gel imaging
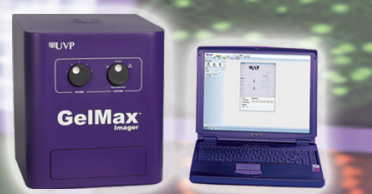

GelDoc-It² Imager

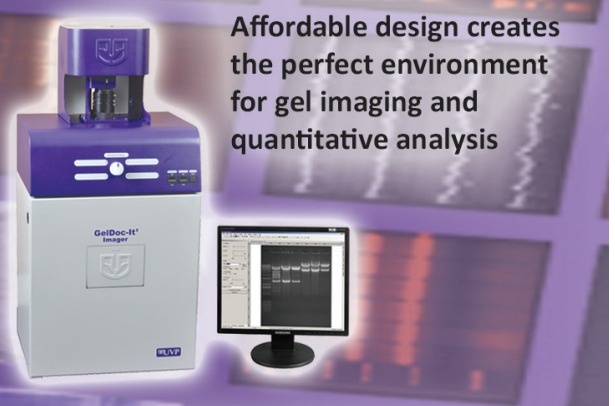

\section{ChemiDoc-It ${ }_{\circledast}^{\text {TS2 }}$ Imager}

A single touch captures vivid gel and blot images for quantitative analysis

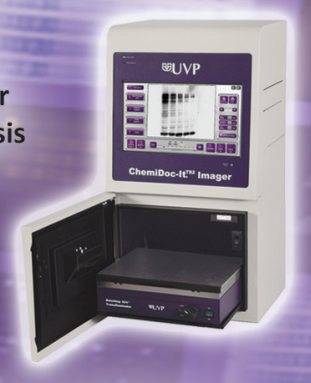

\section{BioSpectrum ${ }^{\circledast}$ Imager}

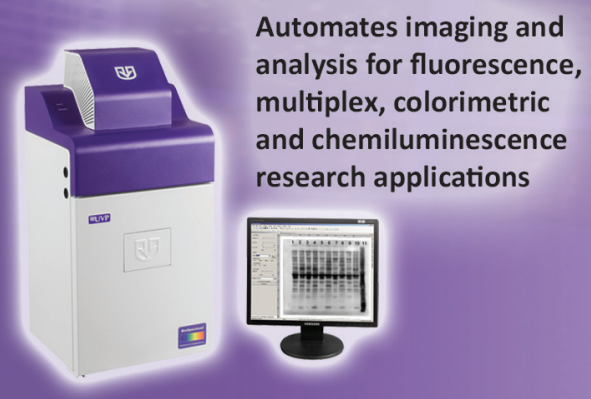

Choose the UVP Biolmaging System solution to match your application and budget needs.

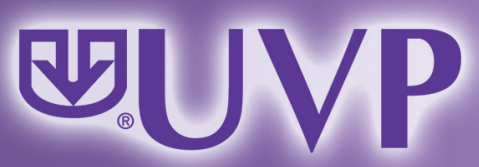

UVP, LLC I USA: (800) 452-6788

Cambridge UK: $+44(0) 1223-420022$

Go to: uvp.com/effortless
Is the protein you are looking at endogenous or recombinant? If it is a tagged recombinant protein, its mobility will be different from the natural version.

You might also try searching the literature for Western blots of your protein to see what size it runs in those blots. If your protein is migrating differently from theirs, see what lysis buffers they use and consider changing your detergent. Also, $12 \%$ acrylamide is too high for a 150 $\mathrm{kDa}$ protein.

A The membrane protein may not be fully denatured under those conditions. I work with porin, which requires boiling in phenol before it can run at its correct molecular weight. Membrane proteins are difficult to work with because the hydrophobicity of their transmembrane regions leads to aggregation. If you run a compact aggregated form of the protein on a gel, it will run faster than the fully denatured protein and will show a band that appears to be lower in apparent molecular weight. You might try some more stringent denaturation steps such as those used for porin to see what happens.

A Does the band run at the correct size for other species? If it does, one possible cause for the problem is cleavage of an Asp-Pro sequence. This has been reported when denaturing proteins using the conditions you mention, but is more likely to occur if the $\mathrm{pH}$ is a bit too low.

I experienced this with a GFP fusion protein linked to my protein of interest by an Asp-Pro sequence in the cloning vector I used. When I blotted with an antibody against GFP, I saw a band for the fusion protein and another just larger than standard GFP in the same lane. Some of the tagged proteins had been cleaved while others remained intact. If this happened and your antibody does not recognize the second fragment of about $40 \mathrm{kDa}$, you will only see one band.

To avoid this, try preparing more samples with strict attention to the $\mathrm{pH}$ before and during denaturation. You may also use a lower temperature to reduce the chance of cleavage at Asp-Pro sequences. I read that $65^{\circ} \mathrm{C}$ is as good as $95^{\circ} \mathrm{C}$ for denaturation. I tried it and the gels look similar to those run with samples denatured at high temperatures.

A When I work with membrane proteins, I do not denature them. It is best to just add the sample buffer and run the gel.
A Repeated freeze-thaw cycles can cleave proteins. Also, beta-mercaptoethanol in your preparation or loading dye will break disulfide bridges. If the $150 \mathrm{kDa}$ protein is a complex of two different proteins held together by a disulfide bridge, the beta-mercaptoethanol might be causing the problem. Boiling will also separate the two pieces.

A Since you are working with a membrane protein, your choice of extraction buffer is quite important. When working with membrane bound proteins, I use 0.1 M TRIS, $1 \%$ Nonidet P-40 substitute (Sigma, 74385), 0.01\% SDS, $1 \mathrm{\mu g} / \mathrm{mL}$ Aprotinin (Roche, 10236624001), and $0.1 \mu \mathrm{M}$ PMSF. Using a standard RIPA buffer with Triton X-100 and sodium-deoxycholate will not extract lipid based proteins well. This could be because the high detergent concentration prevents micelle formation or solubilizes much more protein, resulting in low concentrations of the target protein. What extraction buffer are you using?

$12 \%$ acrylamide is too high a concentration for such a high molecular weight protein. Try using a $7.5 \%$ gel. These are the correct SDS concentrations for resolving proteins of varying sizes:

$$
\begin{gathered}
5 \%: 60-220 \mathrm{kDa} \\
7.5 \%: 45-120 \mathrm{kDa} \\
10 \%: 25-75 \mathrm{kDa} \\
12 \%: 14.4-65 \mathrm{kDa} \\
15 \%: 6.5-45 \mathrm{kDa} \\
17.5 \%: 5.5-30 \mathrm{kDa}
\end{gathered}
$$

You can resolve a $150 \mathrm{kDa}$ band with a $12 \%$ gel, but when blotting, very little of the protein will move out of the gel onto the blot. If you want to verify this, stain the blot with Ponceau $S$ and the gel with Coomassie blue after transfer; that will allow you to see the efficiency of the transfer.

I suspect that your protein either was not extracted properly or that it never left the gel. When you repeat with a different extraction buffer and lower concentration gel, you may see a band at $150 \mathrm{kDa}$ as well as at $110 \mathrm{kDa}$. You would then need to prove by protein sequencing that this is fragment of the target protein and not simply cross-reactivity of an invalid antibody.

\section{Selected by Kristie Nybo, Ph. D. 冝}

BioTechniques 53:23-24 (July 2012) doi: 10.2144/000113887

To purchase reprints of this article, contact:biotechniques@fosterprinting.com 